\title{
CHRONIC SUPPURATIVE OTITIS MEDIA: SURGICAL AND RADIOLOGICAL CORRELATION
}

\author{
By \\ Ahmed A. El-Degwi* MD, Ahmed A. Elasfour* MD, \\ Mohamed M. El-Shaer*, MD, Khalid Mokbel*, MD, \\ And Saleh El-Esawey ${ }^{\star \star}$, MD.
}

\begin{abstract}
From
ENT* Department, and Radiology** Department, Mansoura University.
\end{abstract}

\begin{abstract}
Tympanomastoidectomy operations were done for 63 patients with active chronic suppurative otitis media (CSOM). Preoperative computed tomography (CT) scanning and magnetic resonance imaging (MRI) were performed for all. The CT findings of abnormal soft tissue density associated with bone erosion were $100 \%$ specific to the surgical findings of cholesteatoma. Fifty percent (27/54) of patients that had abnormal soft tissue on CT scan in attic area were accompanied by osseous necrosis of malleus head and incus body. It was not possible to diagnose or exclude cholesteatoma on the basis of CT findings alone. CT and MRI were absolutely specific $(100 \%)$ in detection of soft tissue in attic area, mesotympanum, and mastoid cavity but less sensitive in differentiation of cholesteato-
\end{abstract}

ma from any other soft tissue masses in chronic ear disease. There was a positive significant correlation between CT (or MRI) findings when compared to the surgical findings $(P<0.05)$. Also $C T$ and MRI were significantly correlated $(P<0.05)$. Hyperintensity in $T 1 w$ images makes the diagnosis of lateral sinus thrombophlebitis more conclusive. In our series both CT and MRI were of parallel sensitivity in diagnosing brain abscess with actual cavities.

\section{INTRODUCTION}

High resolution computed tomography $(C T)$ has played a pivotal role in temporal bone imaging since the early 1980s. Although the appearance of magnetic resonance imaging (MRI) technology with its superior demonstration of fluid containing spaces of the inner ear and the posterior fossa MANSOURA MEDICAL JOURNAL 
256 CHRONIC SUPPURATIVE OTITIS MEDIA: SURGICAL etc...

structures, CT continues to offer distinct advantages for evaluation of conductive hearing loss, and petrous apex lesion. This is truly appreciated by skilled interpretation (1)

The foundation of mastoid surgery for cholesteatoma has traditionally been a thorough knowledge of the anatomy and familiarity with landmarks, constant alertness to detect unsuspected complications and the experience to tailor the surgery to the pathology encountered. Whilst not indispensable, CT scanning is a useful adjunct whose potential predictive value is only truly appreciated by skilled interpretation (2)

MRI can be of significant benefit in characterizing expansile lesions of the petrous apex. MRI can be of particular help in distinguishing congenital cholesteatoma from cholesterol granuloma. Furthermore, with the advent of faster high-resolution scanning techniques, MRI can define precise spatial relationships of these masses with the middle and inner ear structures, internal carotid artery, jugular vein, and other structures of the skull base. MRI scanning is sensitive in detecting intracranial extension of these masses. MR angiography can provide additional information regarding the relationship of these masses with adjacent vascular structures and confirm patency of the adjacent vessels. In the postoperative period, MR scanning can help evaluate for complete removal, complication, recurrence, or formation of complicating granulation tissue (3).

As surgery is the only definitive treatment for chronic suppurative otitis media (CSOM), and for several reasons, including low cost and radiation dose, the surgeons have to decide to employ preoperative CT or MRI in every case or not. Nevertheless, many experienced otologic surgeons seldom use CT scanning in their patients with chronic otitis media arguing that the nature and pathology will become evident during surgical dissection. Other surgeons frequently employ preoperative CT scanning in an effort to identify and define potentially dangerous pathologic entities, which might be more safely handled with prior knowledge. MRI is currently encroaching all aspects of temporal bone imaging and can be used as a diagnostic tool of cholesteatoma or any other soft tissue lesion present inside the middle ear cleft (4) 
The intent of this study is to develop criteria for the selective clinical use of CT scanning in CSOM and to point out certain limitations and pitfalls in CT evaluation of such cases, and to find out the exact demand of MRI $i_{\text {. }}$ such cases and its validity in differentiating cholesteatoma from any other soft tissue pathology present in the middle ear cleft.

\section{METHODOLOGY}

A series of 63 patients had CSOM were conducted in the study. Thirtynine had a non-complicated disease, and 24 had clinical manifestations of complications. The whole series were exposed to preoperative CT and MRI.

The 63 cases were collected nonconsecutively from the otology outpatient clinics at Mansoura university hospitals from September 1998 to September 2000. Patients had active CSOM with no previous history of mastoid surgery were enrolled in the study. Patient's consent for radiological and surgical procedures was mandatory. One team of surgeons did all operations. Patients had a generally debilitated diseases, had a local specific disease in the middle ear cleft, or had a contraindication for anaesthesia were excluded from the study. Com- plete history taking, and thorough otolaryngological examination were conducted before any radiological or surgical interference.

CT scans (Philips tomoscan 350) were performed using $1.5 \mathrm{~mm}$ contiguous section through the temporal bone. The majority of scans were obtained in the direct coronal orientation with the patient lying prone. Axially oriented scans were employed when intracranial pathology was suspected or when the patient could not tolerate the position necessary for direct coronal views. MRI was carried out by using $1.5 \mathrm{~T}$. Philips Gyroscan 15 system. GdDtpa $0.1 \mathrm{mmol} / \mathrm{kg}$ body weight was used as a contrast media.

Tympanomastoidectomy operations were done for all patients and extra work was directed to the complications accordingly. Complete operative data were collected on the nature and location of soft tissue masses, the status of the ossicles, and the presence or absence of bone erosion. Pathological confirmation of the clinical diagnosis was obtained. Radiographs were reviewed with particular reference to those anatomic structures important in the preoperative evaluation of CSOM and without 
knowledge of the operative findings.

The collected data was summarized, analyzed and evaluated using the SPSS statistical package. The Chi-Square test was selected to correlate the different study groups. Significance at 0.05 level was estimated to test the null hypothesis.

\section{RESULTS}

The patients included in this study were 34 males and 29 females with age range from $8-67$ years old (mean $=41.8 \pm 8.7$ ). All patients suffered from intermittent aural discharge associated with long-standing tympanic membrane perforation. The cases were classified as: 39 noncomplicated cases, and 24 complicated cases (ten had mastoid abscesses, five had facial paralysis, two had LSCC fistulae, five had brain abscesses, one had extradural abscess and the last one had lateral sinus thrombophlebitis).

CT findings revealed that the attic area bore the main brunt in having the pathological soft tissue in cases of chronic suppurative middle ear infection (54/63), followed by the mesotympanum (43/63), the hypotympanum (35/63), and the mastoid cavity (28i 63) (Table 1). MRI findings revealed soft tissue masses in $49 / 63$, $44 / 63,38 / 63$, and $26 / 63$ in the attic area, the mesotympanum, the hypotympanum, and the mastoid cavity respectively. T1w images appeared hypointense in $18,17,13$, and 11 respectively, while the remaining 30 , 27,25 and 15 appeared of intermediate intensity. There was only one case of the diagnosed soft tissue in the attic area showed hyperintensity in T1w images and surgically proven to be a cholesterol granuloma. T2w images showed hyperintensity for all soft tissue masses that were shown of hypointensity and intermediate intensity by $T 1 w$ images. Lateral sinus thrombophlebitis also showed high signals in T1w images.

Surgical exploration revealed pathological soft tissue in (55/63), (49/ $63),(32 / 63)$ and $(30 / 63)$ in the same order of frequency. During surgery, extension to the adjacent anatomical areas within the middle ear cleft were noticed in more than one third of attic soft tissue (19/55), in near one fifth of mesotympanum soft tissue (10/49), and in less than one sixth in hypotympanum (5/32). Localized soft tissue in the mastoid cavity was noticed in only two cases, while the remaining 28 
cases were coming from the adjacent areas bearing the pathology (Table 11). Differentiation between different pathological types of soft tissue was verified only during surgical exploration. Cholesteatoma was the commonest in the attic area (41/ $55)$ and the least in mesotympanum (21/49) (Table 1). Granulation tissues were present in (13/55), and only one case showed cholesterol granulo$\mathrm{ma}$ in the attic area, while in mesotympanum the granulation tissues account for $26 / 49$, and the remaining two cases showed mucosal oedema (polypi)

Sensitivity and specificity of CT and MRI in diagnosis of soft tissue in middle ear cleft are shown in table III. There was a positive significant correlation (Pearson coefficient) between $\mathrm{CT}$ (or MRI) findings and surgical findings $(P<0.05)$ and this is represented by regression line demonstrated in (Fig. 5\&6). Also there was a positive significant correlation between $C T$ and MRI $(P<0.05)$.

CT diagnosis of ossicles showed absence of malleus head and incus body in 42 cases, absence of long process of incus in 49 cases, and ab- sence of stapes in 58 cases. However, surgical exploration revealed their absence (necrosis) in 27, 47, and 8 cases respectively. Specificity of CT in diagnosis of absent ossicles was $70.6 \%, 88.9 \%$, and $52.4 \%$ respectively. MRI was not evaluated in detection of ossicular absence

Cholesteatoma was associated in most of the cases as follow: $21 / 27$, $39 / 47$, and $8 / 8$ respectively.

Surgical exploration revealed ten cases having erosion in the dural bone plate, two cases having defect in the lateral semicircular canal and five cases having defect in the facial canal. Cholesteatoma was present in all of them. CT was positive in eight, one and three cases respectively (Fig. 1\&2).

MRI of the ten postauricular abscesses encountered in the series showed intermediate intensity in $\mathrm{T} 1 \mathrm{w}$ images and hyperintensity in T2w images. Same MRI intensities were noticed in cases of temporal lobe abscess (Fig. 3) and posterior fossa abscess. Hyperintense signals was noticed in both T1w and T2w images in case of lateral sinus thrombophlebitis (Fig. 4). 
Table I. CT findings versus surgical findings in soft tissue pathology in MEC*

\begin{tabular}{|l|c|l|llll|}
\hline Findings & \multicolumn{2}{|l|}{ CT } & MRI & Surgical & & \\
\hline & Number & Number & Number & $\mathrm{C}^{*}$ & G $^{*}$ & Both \\
\hline Attic area & 54 & 49 & 55 & 41 & 12 & 2 \\
\hline Messtympanum & 43 & 44 & 49 & 21 & 25 & 3 \\
\hline Hypotympanum & 35 & 38 & 32 & 20 & 12 & 0 \\
\hline Mastoid & 28 & 26 & 30 & 17 & 7 & 6 \\
\hline
\end{tabular}

MEC: Middle ear cleft, C: Cholesteatoma, G: Granulation tissue and B: Both.

Table II. Surgical extension of soft tissue.

\begin{tabular}{|l|c|l|l|l|}
\hline Soft tissue & Attic area & Mt.* & Ht.* & Mastoid \\
\hline Localized & 36 & 39 & 27 & 2 \\
\hline Extended & 19 & 10 & 5 & 28 \\
\hline Surgical \# & 55 & 49 & 32 & 30 \\
\hline CT\# & 54 & 43 & 35 & 28 \\
\hline
\end{tabular}

Mt.: Mesotympanum, Ht.: Hypctympanum.

Table III. Sensitivity and specificity of CT and MRI diagnosis of soft tissue according to site

\begin{tabular}{|l|l|l|l|l|}
\hline & CT & CT & MRI & MRI \\
\hline & Sensitivity\% & Specificity\% & Sensitivity\% & Specificity\% \\
\hline Attic area & 98.2 & 100 & 90 & 100 \\
\hline Mesotympanum & 89.1 & 100 & 90.7 & 100 \\
\hline Hypotympanum & 100 & 91.2 & 100 & 83.8 \\
\hline Mastoid & 93.8 & 100 & 88.2 & 100 \\
\hline
\end{tabular}




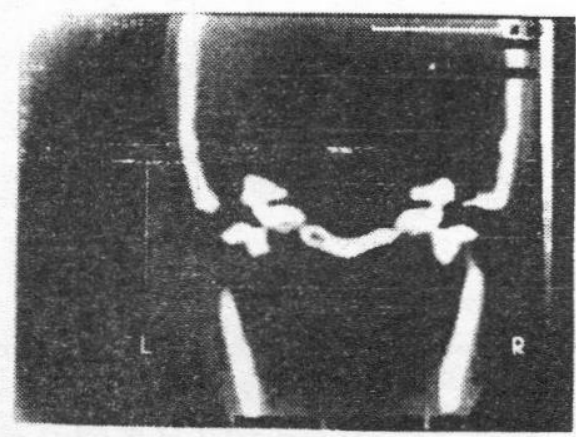

Fig. 1 : Cororial CT showing soft tissue filling middle ear cavity with defect in the dural plate.

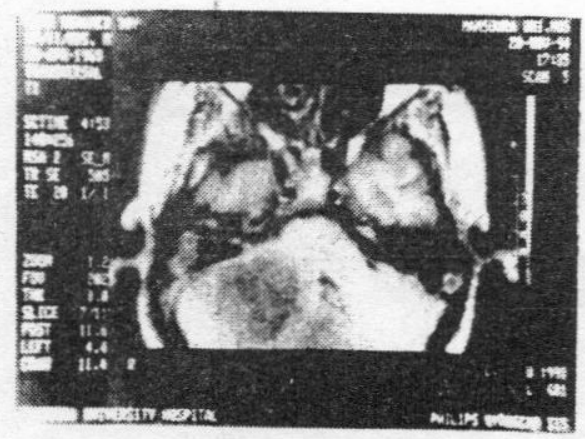

Fig. 3 : MRI, T1W image showing temporal lobe abscess of inter mediate signal.

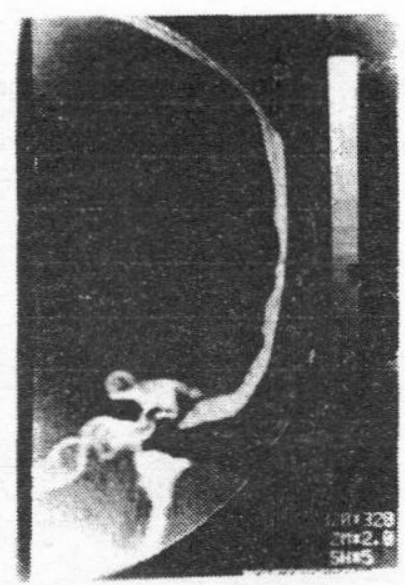

Fig. 2 : Coronal CT showing soft tissue in the attic area with fistula in the lateral SCC.

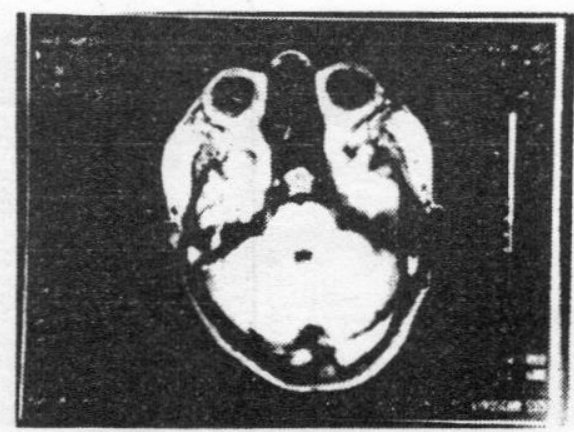

Fig. 4 : MRI, T1 W image showing lateral sinus thrombophlebitis with hyperintense signal.

MANSOURA MEDICAL JOURNAL 


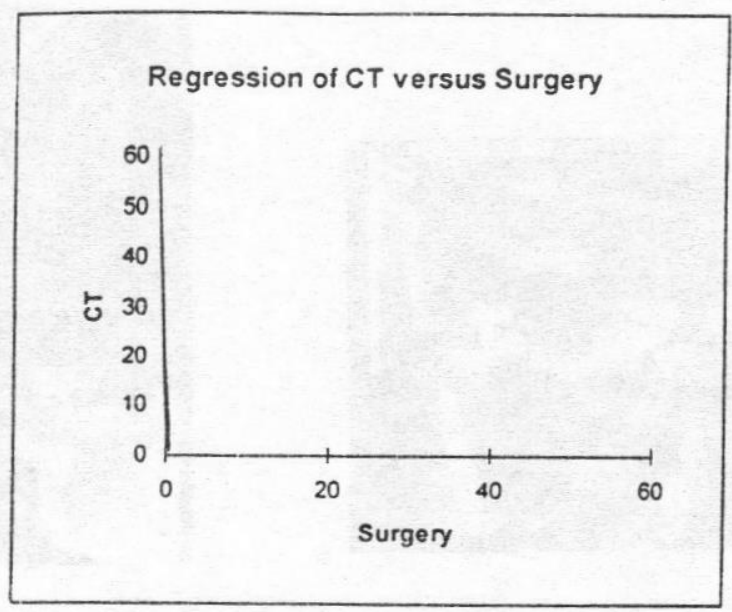

Fig. 5 : Regression of CT versus Surgery

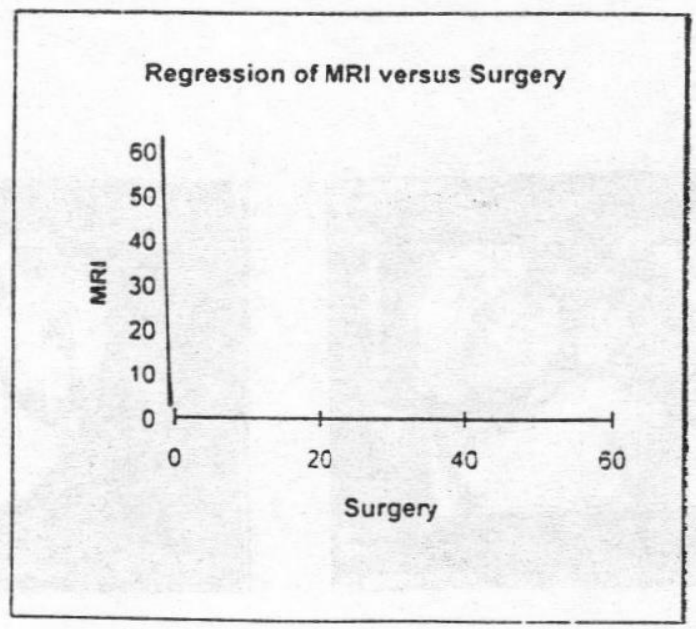

Fig. 6 : Regression of MRI versus Surgery 


\section{DISCUSSION}

CT of chronically draining ears demonstrated abnormal soft tissue densities in the middle ear cleft in most cases. The greatest was in the attic area $54 / 63(85.7 \%)$ (Table 1 ). However, if this soft tissue mass was not associated with bone erosion, it was not possible to discern whether or not cholesteatoma was present. This was true regardless of the amount of soft tissue involvement, its anatomic distribution, or its CT determined density.

In cases where soft tissue filled the attic area the radiographic picture was often highly suggestive of tegmen erosion. Actual erosion of the tegmen was present in 10 of $63 \mathrm{pa}$ tients, eight of which were associated with evident bone destruction in CT (Fig. 1). The duration of the disease and the compressing effect of cholesteatoma may play a role in the bone erosion process. In the series LSCC fistulization was suggested in CT in one case only, while surgical exploration revealed two. This was associated with both an uncovering of the canal lumen and a flattening of the SCC bulge normally seen on the medial wall of the antrum (Fig. 2). Surgical exploration showed cholesteatoma in all of these cases.

CT specificity for ossicular diagnosis showed large false positive results especially for stapes superstructures fuliu'ved by malleus, head and incus, body. Specificity was $52.4 \%$ and $70.6 \%$ respectively. The least false positive resuits were noticed for the long process of incus with a higher specificity $(88.9 \%)$. Therefore, we should not rely on CT alone in diagnosis of ossicular necrosis.

MRI was $100 \%$ specific in detection of soft tissue in middle ear cleft with the exception of soft tissue present in the hypotympanum area $(83.8 \%)$. With the exception of cholesterol gramuloma (one case in the series) that showed high signals in $\mathrm{T} 1 \mathrm{w}$ images, it seems difficult to differentiate cholesteatoma from any other soft tissue masses and from the surrounding inflammatory tissues in the middle ear cleft, as these pathological entities showed hypointensity in some cases and intermediate intensity in others (T1w images), and all T2w images appeared hyperintense. These results could be matched with the series of (5) and (6), as they revealed hypointense images, while (7), (8) and (9) stated that cholesteatoma showed 
intermediate intensity in (T1w images). Hyperintensity in T1w images at the course of lateral sinus makes the diagnosis of lateral sinus thrombophlebitis more conclusive.

As regards the radiological diagnosis of ossicular necrosis in the process of chronic ear diseases, the results were not conclusive. Therefore, we have to anticipate by the audiological assessment, and corroborate by the surgical findings.

In our series both CT and MRI were able to diagnose all cases of brain abscess with actual cavities. Although MRI had better characterization and demarcation of the lesions, CT did not miss any of them. MRI may have the superiority in soft tissue interpretation in early stages of brain abscess that were not met in our series.

In straight forward chronic ear disease, as evidenced by the thorough history and examination, it seems to be no need to expose the patient to radiation of CT or burden him financially by the cost of MRI while everything is quite clear especially when the otologist is eminent. If there is any doubt about the nature of the disease or there is any suspicion of malignancies, CT scanning or MRI may be employed in an effort to identify and define the potentially dangerous pathologic entities, which might be more safely handled with prior knowledge.

\section{Conclusions}

1. Abnormal soft tissue associated with bone erosion on CT scan is highly correlated with cholesteatoma.

2. MRI is sensitive in identifying soft tissue abnormalities at the cost of bony structures, while CT has a comparable sensitivity in soft tissue demonstration with absolute specificity for detection of bone destruction.

3. MRI interpretation of pathological soft tissue in chronic ear disease is still controversial in differentiating cholesteatoma from other soft tissue masses.

4. Preoperative CT could be employed in cases of CSOM, while MRI would be reserved for detection of early intracranial insult.

\section{REFERENCES}

1. Alexander $A E$ Jr; Caldemeyer KS, and Rigby P. (1998) : Clinical and surgical application of reformatted highresolution $\mathrm{CT}$ of the tempor- 
al bone. Neuroimaging Clin N Am (3):631-50.

2. Watt, S; Flood, LM, and Clifford, K. (2000) : A systematic approach to interpretation of computed tomography scans prior to surgery of middle ear cholesteatoma. J Laryngol. Otol, 114(4): 24853.

3. Pisanechi, MJ, and Langer, B. (2000) : Congenital cholesteatoma and cholesterol granuloma of the temporal bone: role of magnetic resonance imaging. Mag. Rosen. Imaging, 11(2): 87-97.

4. Swartz, JD., and Harnsberger, $H$. (1992): The middle ear and mastoid in imaging of the temporal bone, 2nd ed. Thieme Verlag, New York P:148-171.

5. Bowes, A., Weiet, R. (1987) : Brain herniation and space occupying lesions eroding the tegmen tympani. Laryngoscope, 97:1172-1181.

6. Martin, N., Sterkers, O. Murat, M. and Hahum, N. (1989a) : Brain herniation into the middle ear cavity MR imaging. Neuroradiology, 31:184186.

7. Valvassori, G., Carter, B. and Mafee, M. (1989a) : Imaging of the head and neck. Stuttgart, Georg. Thieme Verlag. P:75-84.

8. Valvassori, G. (1996) : Imaging of the temporal bone. In Ballenger, J. and Snow J (ED) Otolaryngology, Head and Neck Surgery, 5 th ed. Lea \& Fabger. Philadelphia, vol. 2:P: $798-825$.

9. Phelps, P. (1997) : Radiology of the ear in Scott-Brown's Otolaryngology. Booth, J. (ed) : 6th edition. Butterworth, vol. 3 chapter, 2: P 4-7. 
266 CHRONIC SUPPURATIVE OTITIS MEDIA: SURGICAL etc...

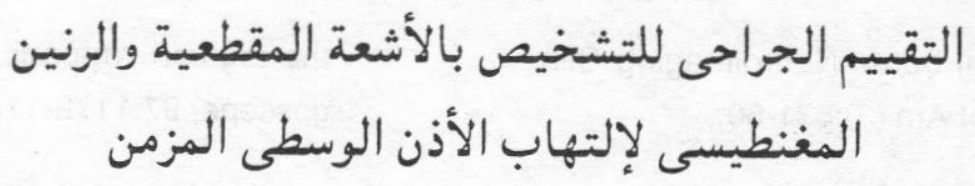

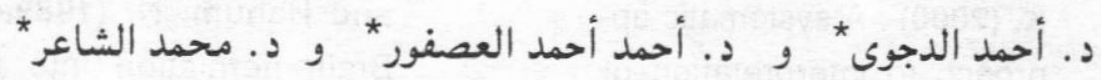

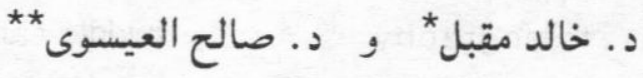

$$
\begin{aligned}
& \text { قسم الأنن والأنف والحنجرة* وقسم الأشعة* } \\
& \text { كلية الطب - جامعة المنصورة }
\end{aligned}
$$

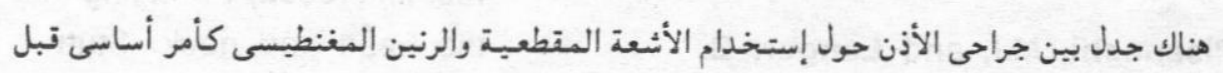

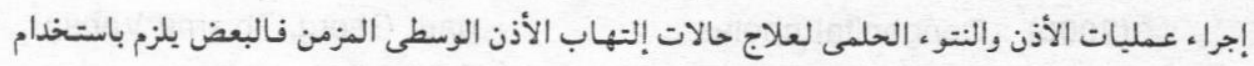

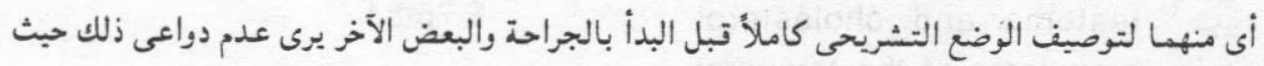
يتم إستكشاف كافة تفاصيل الوضع التشريحى أثناء العملية.

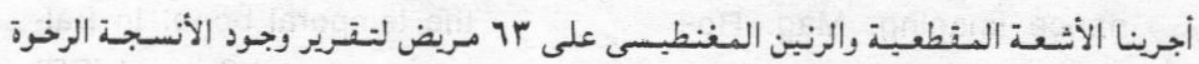

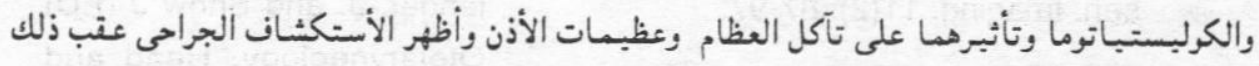

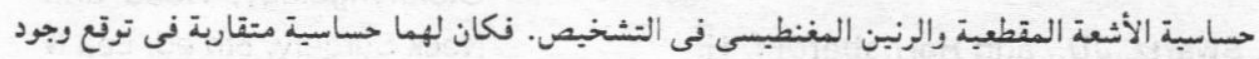

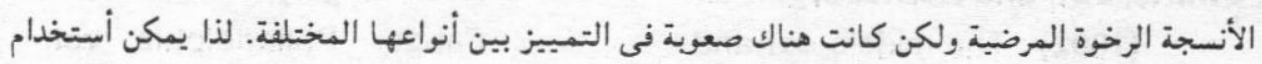

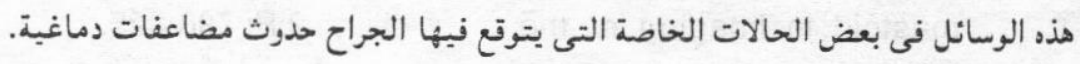

